Research Article

\title{
Logistics Distribution Location Algorithm Based on Improved Imperial Competition Algorithm
}

\author{
Kaituo Su iD \\ Department of Management, Shaanxi Railway Institute, Weinan, Shaanxi 714099, China \\ Correspondence should be addressed to Kaituo Su; sktsxriglxy@163.com
}

Received 24 October 2021; Revised 4 November 2021; Accepted 16 November 2021; Published 8 December 2021

Academic Editor: Le Sun

Copyright (c) 2021 Kaituo Su. This is an open access article distributed under the Creative Commons Attribution License, which permits unrestricted use, distribution, and reproduction in any medium, provided the original work is properly cited.

To solve the problems of warehouse explosion and delay of logistics distribution network under the sudden explosion of demand on "double 11" and "618," this paper proposes a logistics distribution network location algorithm that can consider reliability, green environmental protection, and path optimization. Firstly, the transportation model of logistics distribution network location-route optimization is established. Under the condition that the transportation model satisfies the vehicle path reliability constraint, it can minimize the total cost, including logistics distribution cost, transportation oil consumption, and $\mathrm{CO}_{2}$ emission cost. This paper designs an improved imperial competition algorithm to solve it according to the characteristics of the transportation model. Firstly, the competition mechanism of "United Lian Heng" was introduced in the initial national stage, enhancing the information exchange and retaining the superior population. Secondly, in the process of empire assimilation, we can learn from the colonial rule strategy, which is gradually infiltrated and assimilated by all levels of the country to enhance the development ability of the algorithm. Finally, the algorithm designs a mechanism to judge and jump out of local optimum, so as to avoid "premature" affecting the optimization performance. The rationality of the model and the effectiveness of the improved imperial competition algorithm are verified by simulation experiments of different scales, while the influence of reliability level is analyzed. Experimental results show that the proposed method can effectively solve problems of different scales and maintain stable performance under different reliability levels. Moreover, its algorithm performance is better than that of the standard imperial competition algorithm.

\section{Introduction}

Under the background of regional economic integration and Internet, e-commerce logistics and distribution have developed rapidly. Its characteristics are as follows: (1) facing many customers with small demand, rich varieties, and scattered locations directly, the e-commerce logistics system is complex and costly [1] and (2) customer demand is subject to dynamic fluctuations due to factors such as season, weather, holidays, promotion means, and product life cycle. There is even a sudden surge ("double 11" and "618"). Faced with this kind of disturbance, the resources of each site in the distribution network cannot be accurately balanced. This leads to the phenomena of product explosion and delay, and the risk resistance is poor [2-4]. The distribution network is the foundation of the logistics distribution system.
Reasonable distribution center location and vehicle route planning play an important role in the effective operation of the distribution system [5]. The location routing problem (LRP) integrates both the location problem and the vehicle routing optimization problem [6]. Researchers have studied various LRP problems and their extensions. Michael et al. summarized the related research of LRP in recent years in detail from different angles, including standard LRP, and various extended problems such as multistage LRP, multicycle LRP, and multiobjective LRP [7].

With the enhancement of environmental protection consciousness, people began to pay attention to the environmental pollution caused by $\mathrm{CO}_{2}$ emission in the process of logistics and distribution. Green logistics distribution system design is of great significance to the development of sustainable logistics. There is much research on green LRP. 
In order to minimize the energy cost related to transportation, Benotmane et al. considered the two-level location routing problem. Genetic algorithm and dynamic island model are used to optimize the green LRP problem. The green LRP problem is a combination of the classical location routing problem and the pollution routing problem. This method can minimize energy costs and carbon dioxide emissions [8]. Wang et al. used an ecological package to optimize the location of green logistics. The pickup and delivery of small eco-packages are established through a costminimized synchronous orientation routing model, which is very important for transporting large eco-packages. It is modeled by resource sharing state-time discrete transportation centralized network flow programming in the spatiotemporal network. For the pickup and delivery of small eco-packages, the cost-minimization synchronous guidance positioning path model is adopted [9]. Dukkanci et al. studied the green LRP problem, established a single objective optimization model, minimized the operation cost and emission cost, and considered the time window constraint [10]. Zhang et al. designed a time-dependent green location path problem model with time windows. The model minimizes costs, including the cost of opening warehouses, the cost of starting vehicles, and the cost of fuel consumption [11]. However, the above research does not consider the security problem.

Currently, people pay more and more attention to the security of various systems. Interference inside or outside the distribution system will bring interruption risk to the system, thus affecting the normal operation of the system. Therefore, it is of practical significance to design a safe logistics distribution system. Recently, some scholars have done some research on reliability LRP considering interruption. Raziez et al. designed an LRP model with three types of interruptions in view of the high possibility of interruption of route capacity. According to the NP-hard property of the model, a genetic algorithm is proposed to solve the large-size problem [12]. Badakhshian et al. considered labor interruption or failure caused by bad weather conditions and designed a reliable logistics network by strengthening existing facilities and defining standby facilities to avoid interruption. They proposed a column generation method to model and solve all three logistics problems [13]. Considering the interruption risk of the distribution center, Huang et al. studied the reliable fourthparty logistics routing problem in network design. They established a scenario-based integer programming model to locate the distribution center and minimize the total cost of the proposed problem [14]. In order to make the network resist interruption, Dehghan et al. added interruption in the design stage of the network to reduce the impact of the disaster on LRP. They put forward a mixed-integer programming model. In the supply chain distribution network, the model formulated the reliable capacity positioning path problem with synchronous delivery and service delivery [15]. However, so far, the LRP problem considering the reliability of $\mathrm{CO}_{2}$ emission has been less studied.

Considering reliability and green environmental protection, this paper studies the location path optimization problem of reliable green logistics distribution in order to deal with the warehouse explosion and delay of logistics distribution network under the sudden demand explosion of "double 11" and "618." Different from the existing research on LRP, this paper calculates the fuel consumption and $\mathrm{CO}_{2}$ emission cost based on the network for transport and environment (NTM) method and defines the vehicle routing reliability. This algorithm aims at minimizing the total cost and establishing the logistics distribution location routing optimization model with vehicle routing reliability constraints, designing an improved imperial competition algorithm to solve the transportation model. The improved algorithm explores and solves the problems that the original imperial competitive algorithm (ICA) is greatly influenced by the initial country, lacking effective information interaction between empires. The algorithm is easy to be collected and can be collected early. Finally, the rationality of the model and the effectiveness of the algorithm are verified by experiments.

\section{Problem Description and Model Establishment}

As shown in Figure 1, the logistics distribution network includes distribution centers, customers, distribution vehicles, and distribution lines. Starting from the distribution center, vehicles are distributed by different customers in turn and then returned to the distribution center. In reality, due to the influence of uncertain factors, distribution centers and transportation routes may be interrupted. The interruption will affect the normal operation of the distribution system. In addition, oil consumption and $\mathrm{CO}_{2}$ emission during transportation will cause environmental pollution. The problem of location-route optimization of reliable green logistics distribution can minimize the total logistics cost, fuel consumption, and $\mathrm{CO}_{2}$ emission costs by selecting and opening distribution centers and optimizing the driving routes of different vehicles and at the same time satisfy the constraints of vehicle route reliability.

The model assumes the following conditions. (1) The location, quantity, and demand of customer points are known. (2) The location, quantity, opening cost, capacity, and interruption probability of distribution centers are known. (3) The distance between any two points and the probability of interruption are known. (4) The types of vehicles are different and the number is known, and the fixed operation cost, transportation capacity, transportation cost per unit distance, empty and full fuel consumption of each vehicle are known. (5) At any customer point, only one car must pass. (6) Each vehicle can only start from one distribution center and return to the distribution center. Each distribution center allows multiple vehicles to exit and enter.

2.1. Symbols and Variables. The model symbols are defined as follows:

I: collection of customer points.

$J$ : collection of distribution centers.

$O=I \cup J$.

$S$ : vehicle collection. 


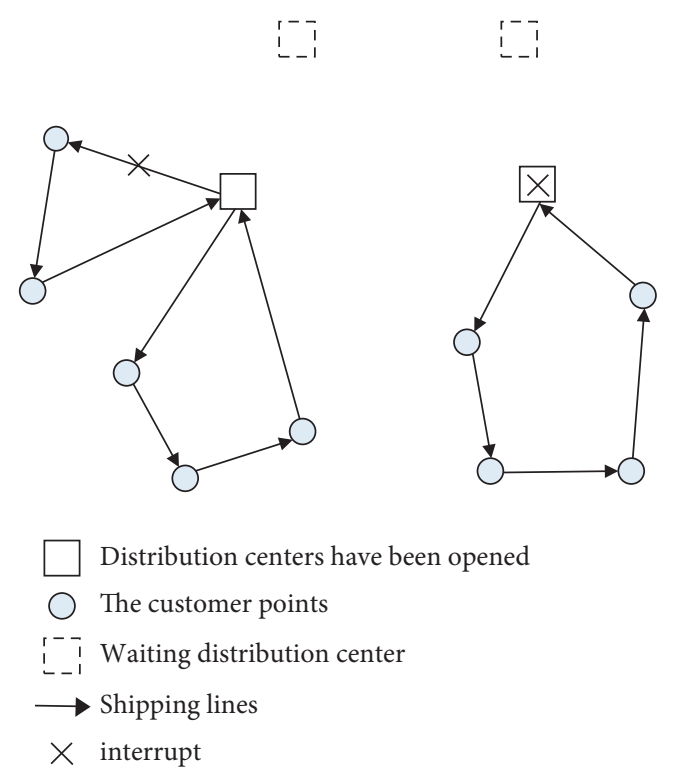

FIGURE 1: Distribution network system.

$D_{i}$ : the demand of customer point $i \in I$.

$R_{j}$ : fixed opening cost of distribution center $j \in J$.

$A_{j}$ : the capacity of distribution center $j \in J$.

$P_{j}$ : the interruption probability of distribution center $j \in J$.

$H_{s}$ : fixed operating cost of vehicle $s \in S$.

$T_{s}$ : transport capacity of vehicle $s \in S$.

$T C_{s}$ : transportation cost per unit distance of vehicle $s \in S$.

$\mathrm{FC}_{s}^{\text {empty }}$ : empty fuel consumption of vehicle $s \in S$.

$\mathrm{FC}_{s}^{\text {fulll }}$ : full-load fuel consumption of vehicle $s \in S$.

$e$ : cost coefficient of oil consumption and $\mathrm{CO}_{2}$ emission.

$D_{i l}:$ the distance from point $i \in O$ to point $l \in O$.

$p_{i l}$ : the interruption probability of the line between point $i \in O$ and point $l \in O$.

$\tau$ : operating period $(\tau=365)$.

Decision variables are defined as follows:

$$
u_{i l k}= \begin{cases}1, & \text { vehicle } s \in S \text { from } i \in O \text { to } l \in O, \\ 0, & \text { else, }\end{cases}
$$

$v_{j k}= \begin{cases}1, & \text { vehicle } s \in S \text { to the distribution center } j \in J, \\ 0, & \text { else, }\end{cases}$

$$
w_{j}= \begin{cases}1, & \text { Open a distribution center } j \in J, \\ 0, & \text { else. }\end{cases}
$$

$f c_{i l k} \geq 0$ indicates the traffic volume of vehicle $s \in S$ between point $i \in O$ and point $l \in O$.

Let $U=\left\{u_{i l k} \mid \forall i, l \in O, s \in S\right\}, V=\left\{v_{j k} \mid \forall j \in J, \forall s \in S\right\}$, $W=\left\{w_{j} \mid \forall j \in J\right\}$.
2.2. Optimization Model. Based on the above symbols and variable definitions, a reliable green logistics distribution network location-path model is established as follows:

$$
\begin{aligned}
\min f(U, V, W)= & \sum_{j \in J} R_{j} w_{j}+\sum_{s \in S} \sum_{j \in J} H_{s} v_{j s} \\
& +\tau\left(\sum_{i \in O} \sum_{l \in O} \sum_{s \in S} \mathrm{TC}_{s} D_{i l} u_{i l s}+\sum_{i \in O} \sum_{l \in O} \sum_{s \in S} \mathrm{FC}_{i l s} D_{i l} u_{i l s} e\right),
\end{aligned}
$$

$\prod_{j \in J}\left(1-P_{j}\right) v_{j s} \prod_{i \in O} \prod_{l \in O}\left(1-p_{i l}\right) u_{i l s} \geq \alpha, \quad \forall s \in S$

$\sum_{i \in I} u_{j i s}-v_{j s}=0, \quad \forall j \in J, \forall s \in S$,

$$
\sum_{s \in S} \sum_{i \in I} u_{j i k}-w_{j} \geq 0, \quad \forall j \in J,
$$

$$
\sum_{i \in I} u_{j i k}-w_{j} \leq 0, \quad \forall j \in J, \forall s \in S,
$$

$$
\sum_{l \in O} u_{l i s}-\sum_{l \in O} u_{i l s}=0, \quad \forall i \in O, \forall s \in S,
$$

$\sum_{i \in M} \sum_{i \in M} u_{i l s} \leq|M|-1, \quad \forall M \subseteq I,|M| \geq 2, \forall s \in S$,

$$
\begin{gathered}
\sum_{j \in J} \sum_{i \in I} u_{j i s} \leq 1, \quad \forall s \in S, \\
\sum_{s \in S} \sum_{l \in O} u_{i l s}=1, \quad \forall i \in I, \\
\sum_{s \in S} u_{j l s}=0, \quad \forall j, l \in J, \\
\sum_{i \in I} \sum_{l \in O} d_{i} u_{i l s} \leq T_{s}, \quad \forall s \in S, \\
\sum_{s \in S} \sum_{i \in I} \sum_{l \in O} d_{i} u_{i l s} v_{j s} \leq A_{j}, \quad \forall j \in J, \\
\sum_{s \in S} \sum_{l \in O} c_{l i s}-\sum_{s \in S} \sum_{l \in O} f c_{i l s}=d_{i}, \quad \forall i \in I, \\
d_{i} u_{i l s} \leq f_{i l s} \leq\left(T_{s}-d_{l}\right) x_{i l s}, \quad \forall i, l \in O, \forall s \in S, \\
u_{i l s} \in\{0,1\}, \quad \forall i, l \in O, \forall s \in S, \\
v_{j s} \in\{0,1\}, \quad \forall j \in J, \forall s \in S, \\
w_{j} \in\{0,1\}, \quad \forall j \in J, \\
f c_{i l s} \geq 0, \quad \forall i, l \in O, \forall s \in S .
\end{gathered}
$$

The objective function (4) minimizes the total cost, including the opening cost of the distribution center, the 
fixed operation cost of the vehicle, the transportation cost, the transportation oil consumption, and the $\mathrm{CO}_{2}$ emission cost, where $F C_{i l s}$ represents the transportation fuel consumption of vehicle $s$ on the line between point $i \in O$ and point $l \in O$. Formula (5) is the path reliability constraint of the vehicle $s$, where the reliability of the vehicle path is defined as the probability that the path keeps running normally. $\alpha$ is the required reliability level. Constraint (6) indicates that vehicle $s$ is assigned to distribution center $j$. Constraint (7) means that vehicles must leave the distribution center. Constraint (8) means that if the distribution center is not opened, no vehicles will leave. Constraint (9) means that the vehicle enters from a point but exits from that point; that is, the path is guaranteed to be a loop. Constraint (10) avoids the formation of subloops between various customer points. Constraint (11) ensures that each vehicle can only exit from one distribution center at most. Constraint (12) ensures that each customer point must have one and only one vehicle service. Constraint (13) requires no vehicle route between any two distribution centers. Constraint (14) represents the capacity constraint of the vehicle. Constraint (15) indicates the capacity constraint of the distribution center. Formula (16) represents the balance constraint of flow at both ends of the customer point. Formula (17) represents the upper limit and lower limit constraint of line flow between two points. Formulas (18)-(20) are binary variable constraints. Formula (21) is the nonnegative constraint of flow between two points.

\subsection{Calculation of Transportation Oil Consumption. In this} paper, the network for transport and environment (NTM) method is used to calculate the fuel consumption of transport vehicles. Based on the NTM method, the fuel consumption of transport vehicles can be calculated by

$$
\mathrm{FC}(l c)=\mathrm{FC}^{\mathrm{empty}}+\left(\mathrm{FC}^{\text {full }}-\mathrm{FC}^{\mathrm{empty}}\right) \cdot l c,
$$

where $\mathrm{FC}^{\text {empty }}$ is the fuel consumption of unloaded vehicles. $\mathrm{FC}^{\text {full }}$ is the fuel consumption of fully loaded vehicles. $l c$ is the load coefficient.

The transportation fuel consumption $\mathrm{FC}_{i l s}$ of the vehicle between the lines between point $i \in O$ and point $l \in O$ is

$$
\mathrm{FC}_{i l s}=\mathrm{FC}_{s}^{\mathrm{empty}}+\left(\mathrm{FC}_{s}^{\text {full }}-\mathrm{FC}_{s}^{\mathrm{empty}}\right) \frac{f c_{i l s}}{T_{s}} .
$$

\section{Logistics Distribution Location Algorithm Based on Improved Imperial Competition Algorithm}

The location-routing problem of reliable green logistics distribution is an extension of the classical LRP problem. Thus, it is also an NP-hard problem. When the scale is large, it is difficult to solve the problem. Thus an intelligent optimization algorithm is designed. In view of the characteristics of the problem model, this paper designs an improved imperial competitive algorithm (IICA) to solve the problem based on the competitive hegemony of vassal states in the
Spring and Autumn period and the Warring States period in Chinese history. The IICA algorithm explores and solves the problems that the original ICA is greatly influenced by the initial country, the lack of effective information exchange between empires, and the easy early collection and early collection.

3.1. Historical Background of Spring and Autumn Period and the Warring States Period. The Spring and Autumn period (770 BC-221 BC) is also called the Eastern Zhou Dynasty. In the early Zhou Dynasty, the Zhou Dynasty divided the imperial clan and heroes into strategic places or around the royal family to fulfill the duties of screening the royal family and expanding the territory, thus forming thousands of vassal states. At the beginning of the Spring and Autumn period, Ping Wang moved to Luoyang, and Zhou Shi began to decline. Because of the different social and economic conditions, the situation of competing for hegemony among countries in the Central Plains gradually appeared. Relying on powerful political and military forces, big countries constantly embezzled and annexed small countries and strengthened their sphere of influence. Small countries also tried to gain a certain living space by means of reform and merger. After two or three hundred years, to the late Spring and Autumn period, only a few dozen larger vassal states remained. During the Warring States period, the pattern of hegemony was formed until Qin destroyed the six countries and unified the world.

During this historical period, a peculiar diplomatic strategy came into being "uniting Lian Heng." That is, in the process of national struggle, weaker countries unite with each other to resist the invasion of powerful countries in order to survive. Once the resistance failed, they took refuge in the powerful countries in order to protect themselves, forming the "vertical" strategy of "uniting the weak to attack the strong" and the "horizontal" strategy of "uniting the strong to attack the weak."

3.2. Improved Imperial Competition Algorithm. Imperial competition algorithm is an intelligent optimization algorithm based on social and political evolution proposed by Atashpaz-Gargari and Lucas. The algorithm can solve the optimization problem by simulating the competition process of imperialist colonies in human society. The ICA algorithm has been applied to solve problems in different fields, such as traveling salesman problem, static synchronous compensator design problem, flexible job shop scheduling problem, and mixed-flow bilateral assembly line balancing problem. The performance of the algorithm has been verified in application. The basic process of ICA can be summarized into four processes: national initialization, colonial assimilation, imperial competition, and national convergence.

ICA simulates the imperial competition mechanism and realizes the optimization ability by approaching the nonoptimal solution to the optimal solution, updating the position of the optimal solution, and competing for convergence [16]. On that basis of retaining the core idea of 
ICA, IICA proposes three improvement measures, and the specific steps are as follows:

(1) Initialization settings, including the initial number of countries $N_{\text {cou }}$, number of empires $N_{\text {suz }}$, number of colonies $N_{\text {col }}$, movement parameter values $\alpha$, and number of iterations.

(2) The initial countries are randomly generated, and the strong country and the weak country are distinguished according to the normalized power value. The strong countries are directly retained, and the weak countries jointly generate a new country. The size of the power value of the new country will determine whether it will replace the existing powerful country or be eliminated, and the remaining country will enter the imperial competition.

(3) The most powerful $N_{\text {suz }}$ countries served as suzerainties to form empires and occupy a corresponding proportion of colonies. The eigenvectors of each colony in the empire move closer to the eigenvectors corresponding to the suzerain. If the colonial power value is greater than the suzerain in the moving process, other colonies will move to the new suzerain instead.

(4) By calculating the total normalized power value of the empire, the weakest colony of the weakest empire will be occupied by other empires, and the occupation probability is directly proportional to the imperial power. The empire with zero colonies will be eliminated and destroyed.

(5) If the same optimal value appears continuously, judge whether it falls into local optimum, and get out of trouble according to the set jump-out scheme.

(6) Iterate until the set maximum number of iterations is reached.

The flow of the improved algorithm is shown in Figure 2.

3.2.1. Improve the Initial State Generation Mechanism. It is difficult to guarantee the quality and stability of the population because the initial country is randomly generated according to the principle analysis of ICA. Based on the historical situation of the elimination of vassal states in the Spring and Autumn period and the Warring States period, this paper attempts a new initial state generation mechanism.

At the beginning of the algorithm, a large number of countries are randomly generated, which is generally $2 \sim 4$ times the number of ICA countries, so that countries with better quality can be retained through competitive screening, and the complexity and operation efficiency of the algorithm will not be greatly affected. Among them, some countries with strong power are retained, while the remaining relatively weak countries avoid elimination via uniting with other countries to strengthen their power in order to protect themselves. Specific steps are as follows:

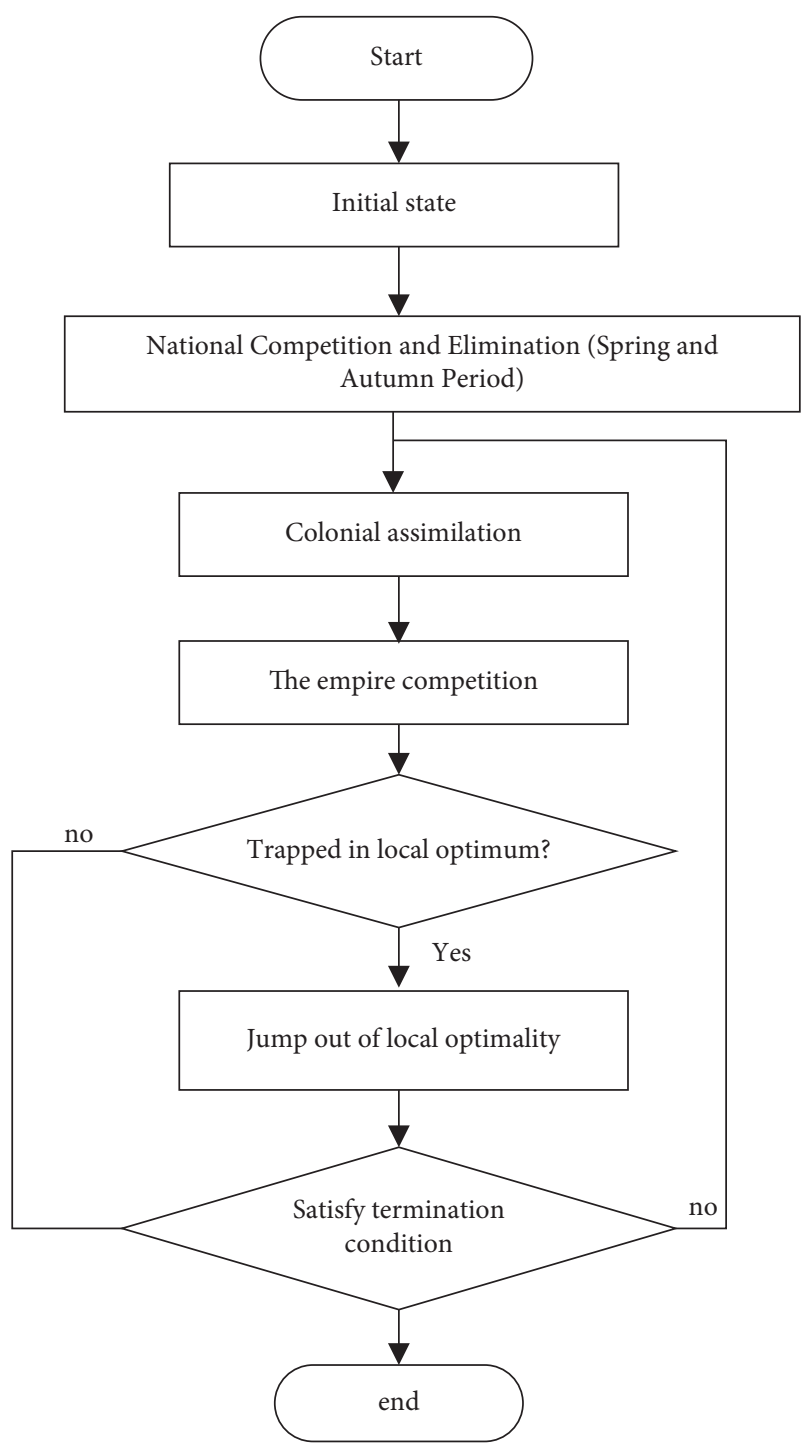

FIgURE 2: The process of IICA process.

(1) A certain number of countries are selected randomly.

(2) Calculate the power of each country and obtain the weight.

(3) Take the location information of each country according to the weight to form and merge into a new country. If the power value of the new country exceeds the retained country, it will be replaced; otherwise, it will be eliminated.

The above competitive screening process enhances the information exchange among countries, retains excellent location information, and forms a better algorithm initial population.

3.2.2. Improve the Way of Empire Assimilation. The process of ICA empire assimilation is realized by moving the colony to the overlord as a whole to improve the way of empire assimilation ICA. The movement parameter $\gamma$ is set to 1.8 to ensure that the colony approaches the overlord from the 
front and back directions. In the real world, colonial assimilation is generally accompanied by infiltration and transformation in politics, economy, and culture. Using this idea for reference, the process of moving the colonial country is changed to the process of moving each feature component to the feature component corresponding to the suzerain country, as shown in Figure 3.

$$
y_{i} \sim U\left(0, \gamma \times d_{i}\right) \cdot i=1,2, \ldots, n .
$$

Each feature component moves independently. If the moving parameter value is $\gamma$, its maximum moving distance is

$$
\sqrt{\left(\gamma d_{1}\right)^{2}+\left(\gamma d_{2}\right)^{2}+\cdots+\left(\gamma d_{n}\right)^{2}}=\gamma d
$$

where $d_{i}(i=1,2, \ldots, n)$ is the distance between each characteristic component of the colony and the corresponding characteristic component of the empire. The value of $\gamma$ should be greater than 1.0 in order to ensure the approach from both sides to the target. After repeated tests and experiments, it is proved that when the value of $\gamma$ is gradually increased from 1.0, the solution accuracy is gradually improved. Different test functions can obtain the best optimization accuracy when the $\gamma$ value is between 1.5 and 1.8. After $\gamma$ is taken as 1.8 , the solution accuracy gradually decreases as the value of $\gamma$ increases. Therefore, for different objective functions, limiting the $\gamma$ value of the movement parameter to the interval between 1.5 and 1.8 can achieve better results. In experiments or practical applications, a simple substitution test can be performed first to clarify the parameter values.

In order to compare the difference between the improved algorithm and the original algorithm in the moving mechanism, Figure 4 shows the difference in search range between the two algorithms in the two-dimensional plane. The search range of ICA is a sector with a radius of $2 d$, while the improved algorithm is a rectangle with the maximum moving range of each component as its side. On the one hand, the independent movement of each feature component enhances the flexibility and randomness, and the development ability is stronger. On the other hand, the rectangular search range is relatively small, which improves the overlap and coverage of search areas when multiple targets are close (Figure 5), and has higher efficiency.

\subsubsection{Increase the Strategy of Jumping Out of Local Optimum.} Aiming at the premature problem of the original ICA, a strategy of jumping out of the local optimum is proposed. If the same optimal value appears many times in succession, it is judged to fall into the local optimum. At this time, the suzerain jumps out of the local optimum according to the selected scheme.

There are many schemes for jumping out of the local optimum. Three schemes are given and tested in this paper. Firstly, take the arithmetic mean of each suzerain as the new moving target point, which is denoted IICA-I in this paper. Secondly, according to the power of each suzerain, the weighted average is taken as a new moving target point,

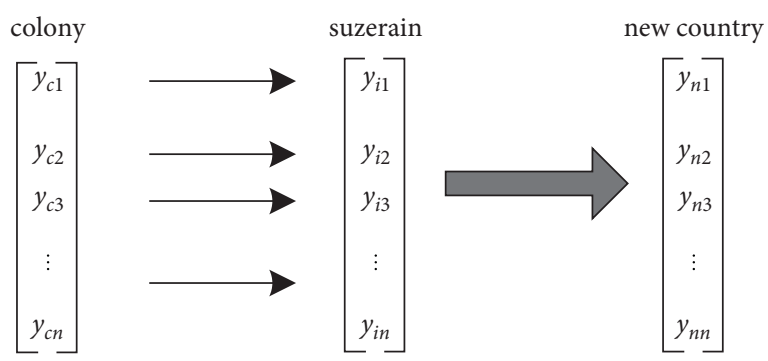

FIGURE 3: Individual movement of feature components.

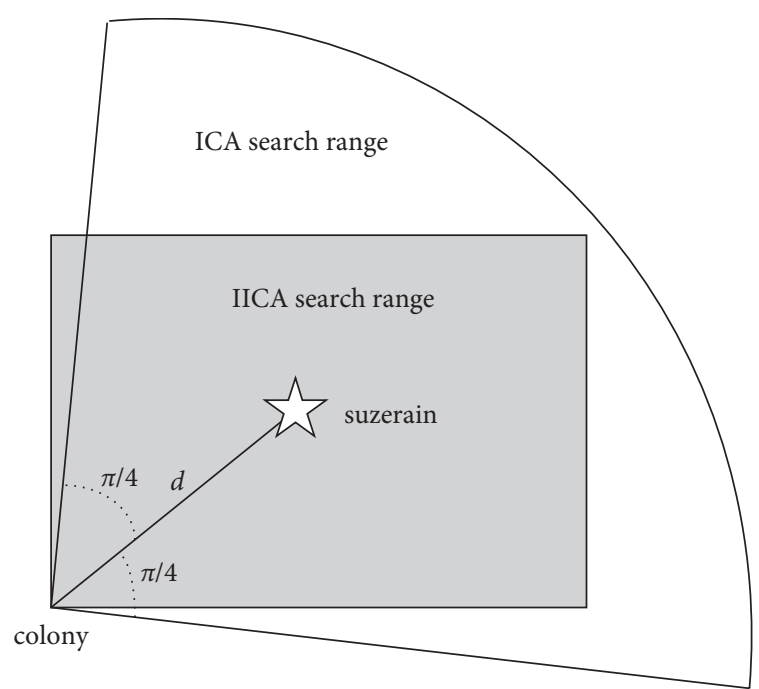

Figure 4: The comparison of the search range.

which is recorded as IICA-II in this paper. Thirdly, a certain percentage of the location information of the suzerain changed randomly, which was set as $10 \%$ in this paper and marked as IICA-III. The new moving target points constructed by the first two schemes all contain the information of other suzerain countries, which enhances the information interaction. The third scheme jumps out of the local optimum through random changes, which improves the population diversity.

3.3. The Development and Exploration Ability of the Improved Algorithm and Their Balance Analysis. ICA is deficient in balancing development and exploration capabilities and lacks effective information interaction. The merger and collapse of empires rapidly reduce population diversity, and the applicability of high-dimensional functions is not strong. In view of the above problems, IICA uses a competitive elimination mechanism to enhance information exchange and retain dominant populations in the initial national stage. After many iterations, the mechanism of jumping out of local optimum is added in the stage of greatly reducing population diversity, which provides a path for the recovery of population diversity. At the same time, the improvement of the assimilation mechanism enhances the flexibility and efficiency of search and improves the development ability without affecting the exploration ability. Therefore, 


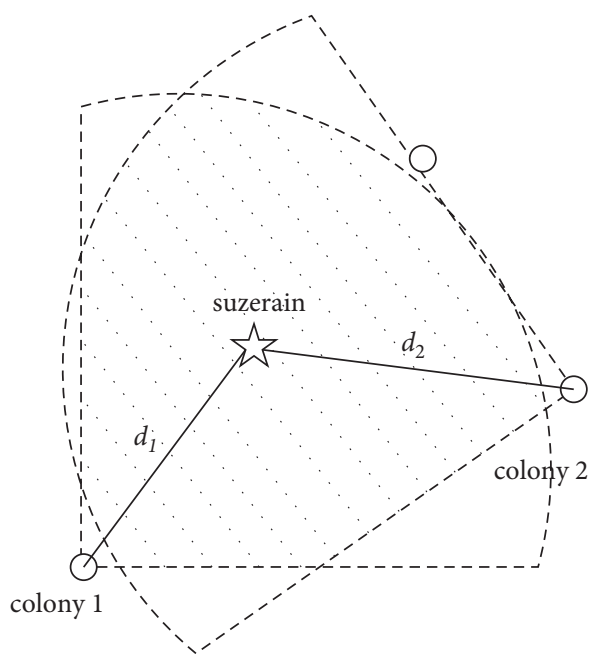

(a)

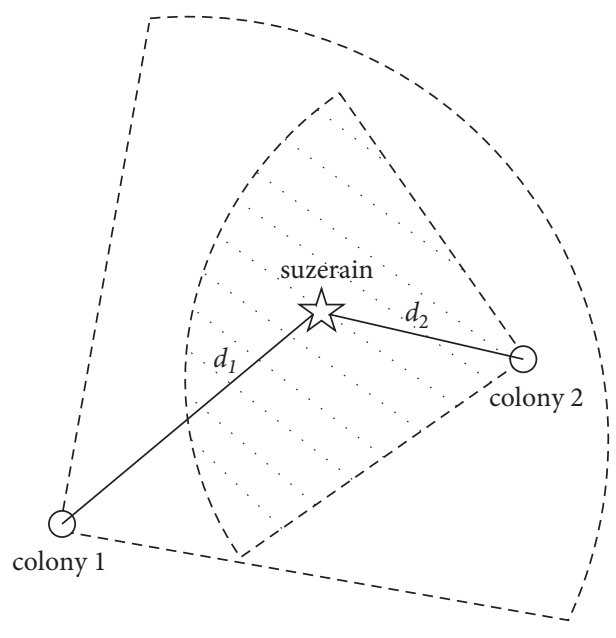

(b)

FIgURE 5: Search area crossing and coverage. (a) Search area crossing. (b) Search area coverage.

TABLE 1: Scale of examples.

\begin{tabular}{lcccc}
\hline Scale & Examples & Number of distribution centers & Number of customers & Number of vehicles \\
\hline \multirow{2}{*}{ Large scale } & 11 & 7 & 70 & 42 \\
& 12 & 8 & 50 & 18 \\
\multirow{2}{*}{ Medium scale } & 13 & 5 & 50 & 30 \\
\hline \multirow{2}{*}{ Small scale } & 14 & 6 & 30 & 34 \\
& 15 & 3 & 30 & 18 \\
\hline
\end{tabular}

compared with ICA, the development and exploration capabilities of the improved algorithm are enhanced simultaneously, and there are more measures to improve the exploration capabilities. The balance between the two algorithms is more reasonable.
3.4. Cost Function. Given an imperialist country or a colonial country, the solution $(U, V, W)$ is obtained by decoding, and the national cost function is calculated according to

$$
C=f(U, V, W)+\varepsilon_{1} \sum_{s \in S}\left(\alpha-\prod_{j \in J}\left(1-P_{j}\right) v_{j s} \prod_{i \in O} \prod_{l \in O}\left(1-p_{i l}\right) u_{i l s}\right)+\varepsilon_{2} \sum_{s \in S}\left(\sum_{i \in I} \sum_{l \in O} d_{i} u_{i l s}-T_{s}\right)^{+}+\varepsilon_{3} \sum_{j \in J}\left(\sum_{s \in S} \sum_{i \in I} \sum_{l \in O} d_{i} u_{i l s} v_{j s}-\mathrm{TC}_{j}\right)^{+} .
$$

Among them, $f(U, V, W)$ is the objective function expression (4), and the path reliability constraint (5), vehicle capacity constraint (14), and distribution center capacity constraint (15) are added to the evaluation function as penalty items. $\epsilon_{1}, \epsilon_{2}$, and $\epsilon_{3}$ are penalty coefficients. ( $)^{+}$means that if the value in brackets is positive, the value will be taken. $\alpha$ represents the level of reliability. $P_{j}$ represents the outage probability of the distribution center. $p_{i l}$ represents interruption probability of the line between point $i \in O$ and point $l \in O . d_{i}$ represents customer demand. $T_{s}$ represents the transportation capacity of the vehicle. $\mathrm{TC}_{j}$ represents vehicle unit distance transportation cost.

\section{Experimental Results and Analysis}

4.1. Experimental Parameter Setting. In order to verify the rationality of the model and the effectiveness of the IICA algorithm, different numerical examples are tested. Table 1 shows the scale of different examples, including the number of distribution centers, customers, and vehicles. The calculated parameters are generated according to the uniform distribution in Table 2 . The algorithm is realized by python programming, and the experimental environment for all tests is a computer with Intel Core i7 $\mathrm{CPU} 2.6 \mathrm{GHz}$ and memory $8 \mathrm{~GB}$ and operating system Windows 10. 
TABle 2: Parameters of calculation examples.

\begin{tabular}{lc}
\hline Parameter & Uniform distribution interval \\
\hline Customer demand $d_{i}$ & {$[100,200]$} \\
The opening cost of the distribution center $R_{j}$ & {$[20000,40000]$} \\
The capacity of the distribution center $A_{j}$ & {$[3000,5000]$} \\
The outage probability of the distribution center $P_{j}$ & {$[0,0.1]$} \\
Fixed operating cost of the vehicle $H_{s}$ & {$[3000,5000]$} \\
The transportation capacity of the vehicle $T_{s}$ & {$[1500,2500]$} \\
Vehicle unit distance transportation cost $T C_{s}$ & {$[1,5]$} \\
No-load fuel consumption of the vehicle $\mathrm{FC}_{s}^{\text {empty }}$ & {$[0.1,0.15]$} \\
The fuel consumption of the vehicle at full-load $\mathrm{FC}_{s}^{\text {full }}$ & {$[0.15,0.2]$} \\
$\mathrm{CO}_{2}$ emission and fuel consumption cost coefficient $e$ & {$[10,15]$} \\
The distance $D_{i l}$ between the point $i \in O$ and point $l \in O$ & {$[1,5]$} \\
Interruption probability $p_{i l}$ of the line between point $i \in O$ and point $l \in O$ & {$[0,0.1]$}
\end{tabular}

TABle 3: Comparison of IICA, ICA, and HS-SA results under different scale examples.

\begin{tabular}{|c|c|c|c|c|c|c|}
\hline Examples & Algorithm & Best value & $\begin{array}{l}\text { Maximum difference } \\
\text { value }\end{array}$ & Average value & $\begin{array}{c}\text { Average deviation rate } \\
(\%)\end{array}$ & Average CPU running time (s) \\
\hline \multirow{3}{*}{ I1 } & IICA & 473292.10 & 495635.29 & 485116.74 & 2.50 & 208.01 \\
\hline & ICA & 508532.16 & 5552807.32 & 542479.12 & 6.68 & 210.27 \\
\hline & HS-SA & 476899.28 & 540145.96 & 509045.71 & 6.74 & 238.20 \\
\hline \multirow{3}{*}{ I2 } & IICA & 481755.47 & 505259.35 & 491528.37 & 2.03 & 202.98 \\
\hline & ICA & 508364.11 & 536836.58 & 528604.79 & 3.98 & 207.08 \\
\hline & HS-SA & 514238.19 & 557061.58 & 532107.68 & 3.47 & 232.94 \\
\hline \multirow{3}{*}{ I3 } & IICA & 297755.56 & 322837.48 & 306812.79 & 3.04 & 132.90 \\
\hline & ICA & 338145.28 & 386614.43 & 369751.38 & 9.35 & 141.73 \\
\hline & HS-SA & 322288.01 & 368362.18 & 341486.69 & 5.96 & 167.97 \\
\hline \multirow{3}{*}{ I4 } & IICA & 250995.19 & 265680.99 & 258108.18 & 2.83 & 135.25 \\
\hline & ICA & 258353.54 & 306575.04 & 281304.55 & 8.88 & 143.92 \\
\hline & HS-SA & 254393.24 & 324192.23 & 273091.02 & 7.35 & 163.14 \\
\hline \multirow{3}{*}{$I 5$} & IICA & 130179.00 & 137153.13 & 134007.02 & 2.94 & 101.50 \\
\hline & ICA & 144109.44 & 157950.78 & 153328.73 & 6.40 & 105.46 \\
\hline & HS-SA & 136060.07 & 151761.65 & 142757.37 & 4.92 & 112.80 \\
\hline \multirow{3}{*}{ I6 } & IICA & 175181.59 & 197875.06 & 180529.97 & 3.05 & 103.24 \\
\hline & ICA & 191526.10 & 239578.56 & 220382.78 & 15.07 & 107.91 \\
\hline & HS-SA & 180761.54 & 219009.74 & 198518.34 & 9.82 & 120.59 \\
\hline
\end{tabular}

4.2. Experimental Results. This algorithm is compared with the existing methods in order to verify the effectiveness of this method. Comparison methods are standard ICA and hybrid harmonic search-simulated annealing (HS-SA) algorithm proposed in [17]. The HS-SA algorithm combines the dynamic value of harmony memory considering the speed and pitch adjustment rate with local optimization technology and combines the idea of probability acceptance rule of simulated annealing to avoid local extreme points.

Firstly, the performance of IICA is tested by solving I1 I6 examples of different scales, and the results are compared with the standard ICA algorithm and HS-SA algorithm. Among them, the reliability level $\alpha$ of all examples is 0.5 . For a fair comparison, the number of initial countries of the IICA algorithm and standard ICA algorithm is 100 , the number of initial empires is 10 , and the maximum number of iterations is 100 . The value of constant $f$ of the IICA algorithm is 1.5. The HS-SA algorithm has a population size of 100 , a maximum number of iterations of 100 .
For each example, each algorithm is run 10 times, respectively. Table 3 shows the results' comparison of the IICA, ICA, and HS-SA algorithms under different scales, including best value, worst value, average value, average deviation rate, and average CPU running time. Among them, the average deviation rate is defined as ((average value - best value of average)/best value) $\times 100 \%$.

It can be seen from Table 4 that the best value, the worst value, the average value, and the average deviation rate of the IICA algorithm are lower than those of the standard ICA algorithm and HS-SA algorithm for examples of different scales. Among them, the average deviation rate of the IICA algorithm varies from $2.0 \%$ to $3.1 \%$. However, the average deviation rate of the standard ICA algorithm varies from $3.9 \%$ to $15.1 \%$, and that of the HS-SA algorithm varies from $3.5 \%$ to $9.8 \%$. It can be seen that the IICA algorithm can effectively solve examples of different scales, and with the increase of problem scale, the IICA algorithm can still maintain good performance. In addition, the average running time of the IICA algorithm is lower because of the 
TABLE 4: Comparison of IICA and ICA results under different reliability levels.

\begin{tabular}{lccccc}
\hline Reliability level & Algorithm & Best value & Worst value & Average value & Average deviation rate \\
\hline \multirow{2}{*}{0.3} & IICA & 263402.63 & 302897.72 & 279755.16 & 6.21 \\
& ICA & 285625.69 & 325442.51 & 314833.18 & 10.23 \\
\hline \multirow{2}{*}{0.4} & IICA & 275002.06 & 330545.00 & 295729.07 & 7.54 \\
& ICA & 294063.73 & 348928.00 & 329603.84 & 12.09 \\
\hline \multirow{2}{*}{0.5} & IICA & 298955.56 & 333039.50 & 307812.79 & 3.96 \\
& ICA & 338145.28 & 376816.45 & 359751.38 & 7.35 \\
\hline \multirow{2}{*}{0.6} & IICA & 326348.99 & 379520.85 & 350530.03 & 3.83 \\
\hline \multirow{2}{*}{0.7} & ICA & 346499.02 & 406936.54 & 380719.97 & 4.81 \\
& IICA & 383600.81 & 440393.86 & 402062.11 & 9.44 \\
\hline \multirow{2}{*}{0.8} & ICA & 429459.97 & 490012.29 & 469998.58 & 5.52 \\
\end{tabular}

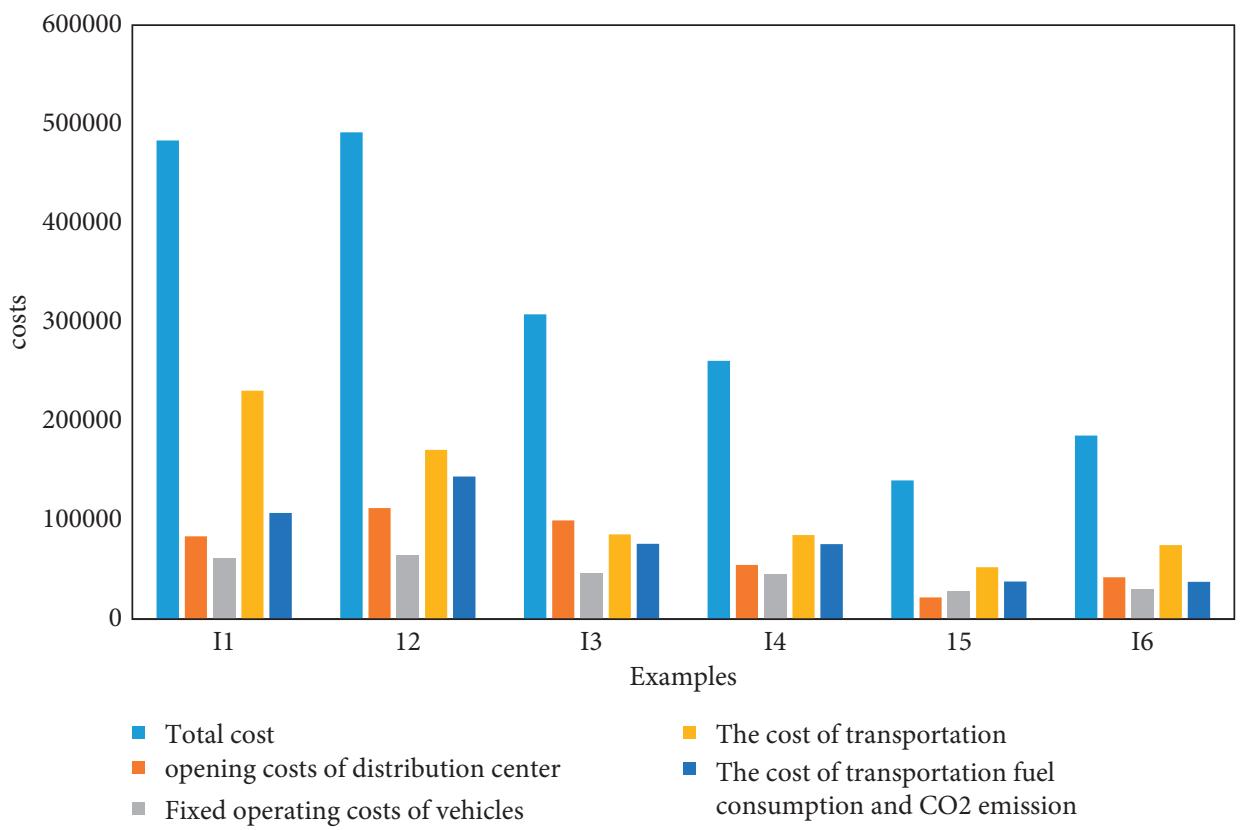

FiguRE 6: Detailed results of examples of different scales.

improved empire assimilation method and the strategy of jumping out of the local optimum.

Figure 6 shows the detailed results of examples I1 I6, including the opening cost of the distribution center, the fixed operation cost of vehicles, transportation cost, transportation oil consumption, and $\mathrm{CO}_{2}$ emission cost. It can be seen from Figure 6 that the larger the scale of logistics distribution, the greater the vehicle operating cost, transportation cost, transportation fuel consumption, and $\mathrm{CO}_{2}$ emission cost, which ultimately leads to an increase in the total cost.

In order to analyze the influence of reliability level $\alpha$ on algorithm performance and optimization decision results, an example I3 is taken as an example. Among them, the reliability level $\alpha$ is $0.3 \sim 0.8$. For different reliability levels, each algorithm is run 10 times, respectively. Table 4 shows the results' comparison between the IICA algorithm and standard ICA algorithm under different reliability. It can be seen from Table 4 that the average deviation rate of the IICA algorithm ranges from $2.9 \%$ to $12.6 \%$, while the average deviation rate of the standard ICA algorithm is between $9.3 \%$ and $16.9 \%$. The best value, the worst value, and the average value of the IICA algorithm are also superior to the standard ICA algorithm. Therefore, the IICA algorithm can still maintain stable performance under different reliability levels, and its solution effect is better than that of the standard ICA algorithm.

\section{Conclusion}

In this paper, the problem of location-route optimization for reliable green logistics distribution is studied, and an optimization model is established to minimize the total cost, including the opening cost of the distribution center, vehicle operation cost, transportation cost, transportation oil consumption, and $\mathrm{CO}_{2}$ emission cost, under the condition of 
satisfying the constraint of route reliability. An improved imperial competition algorithm (IICA) is designed according to the characteristics of the problem. Finally, the performance of the algorithm is tested by a numerical example, and the important parameters are analyzed. The experimental results show that the average deviation rate of the IICA algorithm varies from $2.5 \%$ to $8.3 \%$, which is better than that of the standard ICA algorithm and HS-SA algorithm. IICA algorithm can effectively solve different scale examples. Furthermore, with the increase of the problem scale, the IICA algorithm can still maintain good performance. In addition, with the increase of reliability level, the number of vehicles increases, which increases the vehicle operating cost, transportation cost, transportation fuel consumption, and $\mathrm{CO}_{2}$ emission cost and finally leads to the increase in the total cost. In future research, the reliability of green logistics distribution location-route optimization in a dynamic environment can be further considered.

\section{Data Availability}

The labeled dataset used to support the findings of this study is available upon request to the author.

\section{Conflicts of Interest}

The author declares no conflicts of interest.

\section{References}

[1] Y. Zhong, F. Guo, and Z. Wang, "Coordination analysis of revenue sharing in E-commerce logistics service supply chain with cooperative distribution," Sage Open, vol. 9, no. 3, Article ID 2158244019870536, 2019.

[2] A. V. Barenji, W. M. Wang, Z. Li, and D. A. Z. Guerra, "Intelligent E-commerce logistics platform using hybrid agent based approach," Transportation Research Part E: Logistics and Transportation Review, vol. 126, pp. 15-31, 2019.

[3] K. Zheng, Z. Zhang, and B. Song, "E-commerce logistics distribution mode in big-data context: a case analysis of JD.COM," Industrial Marketing Management, vol. 86, pp. 154-162, 2020.

[4] M. Janjevic and M. Winkenbach, "Characterizing urban lastmile distribution strategies in mature and emerging e-commerce markets," Transportation Research Part A: Policy and Practice, vol. 133, pp. 164-196, 2020.

[5] Z. Ning, X. Hu, Z. Chen et al., "A cooperative quality-aware service access system for social Internet of vehicles," IEEE Internet of Things Journal, vol. 5, no. 4, pp. 2506-2517, 2018.

[6] M. Darvish, C. Archetti, L. C. Coelho, and M. G. Speranza, "Flexible two-echelon location routing problem," European Journal of Operational Research, vol. 277, no. 3, pp. 1124-1136, 2019.

[7] M. Schneider and M. Drexl, "A survey of the standard location-routing problem," Annals of Operations Research, vol. 259, no. 1, pp. 389-414, 2017.

[8] Z. Benotmane, G. Belalem, and A. Neki, "Green optimisation for LRP problem using a genetic algorithm and a dynamic island model," International Journal of Advanced Operations Management, vol. 11, no. 1-2, pp. 46-68, 2019.

[9] Y. Wang, S. Peng, and X. Zhou, "Green logistics locationrouting problem with eco-packages," Transportation Research
Part E: Logistics and Transportation Review, vol. 143, pp. 1-33, 2020.

[10] O. Dukkanci, B. Y. Kara, and T. Bektaş, "The green locationrouting problem," Computers \& Operations Research, vol. 105, pp. 187-202, 2019.

[11] C. Zhang, Y. Zhao, and L. Leng, "A hyper-heuristic algorithm for time-dependent green location routing problem with time windows," IEEE Access, vol. 8, Article ID 83092, 2020.

[12] H. Peng, B. Hu, Q. Shi et al., "Removal of ocular artifacts in EEG-an improved approach combining DWT and ANC for portable Applications," IEEE journal of biomedical and health informatics, vol. 17, no. 3, pp. 600-607, 2013.

[13] M. Badakhshian, Location and location-routing problems with disruption risks, Thesis (PhD), Concordia University, Montreal, Canada, 2019.

[14] M. Huang, L. Dong, H. Kuang, and X. Wang, "Reliable fourth party logistics location-routing problem under the risk of disruptions," IEEE Access, vol. 9, Article ID 84857, 2021.

[15] M. Dehghan, S. R. Hejazi, M. K. Mamaghan, M. Mohammadi, and A. Pirayesh, "Capacitated location routing problem with simultaneous pickup and delivery under the risk of disruption," RAIRO - Operations Research, vol. 55, no. 3, pp. 1371-1399, 2021.

[16] Q. Fang, H. Nguyen, X. N. Bui, and T. T. Nguyen, "Prediction of blast-induced ground vibration in open-pit mines using a new technique based on imperialist competitive algorithm and M5Rules," Natural Resources Research, vol. 29, no. 2, pp. 791-806, 2020.

[17] F. Misni, L. S. Lee, and H. V. Seow, "Hybrid harmony searchsimulated annealing algorithm for location-inventory-routing problem in supply chain network design with defect and nondefect items," Applied Sciences, vol. 10, no. 18, pp. 6625-6647, 2020. 